\title{
Demagnifying gravitational lenses toward hunting a clue of exotic matter and energy
}

\author{
Takao Kitamura, Koki Nakajima, and Hideki Asada \\ Faculty of Science and Technology, \\ Hirosaki University, Hirosaki 036-8561, Japan
}

(Dated: October 9, 2018)

\begin{abstract}
We examine a gravitational lens model inspired by modified gravity theories and exotic matter and energy. We study an asymptotically flat, static, and spherically symmetric spacetime that is modified in such a way that the spacetime metric depends on the inverse distance to the power of positive $n$ in the weak-field approximation. It is shown analytically and numerically that there is a lower limit on the source angular displacement from the lens object to get demagnification. Demagnifying gravitational lenses could appear, provided the source position $\beta$ and the power $n$ satisfy $\beta>2 /(n+1)$ in the units of the Einstein ring radius under a large- $n$ approximation. Unusually, the total amplification of the lensed images, though they are caused by the gravitational pull, could be less than unity. Therefore, time-symmetric demagnification parts in numerical light curves by gravitational microlensing (F.Abe, Astrophys. J. 725, 787, 2010) may be evidence of an Ellis wormhole (being an example of traversable wormholes), but they do not always prove it. Such a gravitational demagnification of the light might be used for hunting a clue of exotic matter and energy that are described by an equation of state more general than the Ellis wormhole case. Numerical calculations for the $n=3$ and 10 cases show maximally $\sim 10$ and $\sim 60$ percent depletion of the light, when the source position is $\beta \sim 1.1$ and $\beta \sim 0.7$, respectively.
\end{abstract}

PACS numbers: 04.40.-b, 95.30.Sf, 98.62.Sb 


\section{INTRODUCTION}

The bending of light was the first experimental confirmation of the theory of general relativity. Nowadays, gravitational lensing is one of the most important tools in astronomy and cosmology. It is widely used for investigating extrasolar planets, dark matter, and dark energy.

Light bending is also of theoretical importance, in particular for studying a null structure of a spacetime. A rigorous form of the bending angle plays an important role in properly understanding a strong gravitational field [1 5] . For example, strong gravitational lensing in a Schwarzschild black hole was considered by Frittelli, Kling, and Newman [1] and by Virbhadra and Ellis [2]; Virbhadra and Ellis [3] later described strong gravitational lensing by naked singularities; Eiroa, Romero, and Torres [4] treated Reissner-Nordström black hole lensing; Perlick [5] discussed lensing by a Barriola-Vilenkin monopole and also by an Ellis wormhole.

One of the peculiar features of general relativity is that the theory admits a nontrivial topology of a spacetime; for instance, a wormhole. An Ellis wormhole is a particular example of the Morris-Thorne traversable wormhole class [6 8]. Many years ago, scattering problems in such spacetimes were discussed (for instance, [9, 10]). One remarkable feature is that the Ellis wormhole has zero mass at the spatial infinity, but it causes light deflection [9, 10]. Moreover, gravitational lensing by wormholes has been recently investigated as an observational probe of such an exotic spacetime [5, 11 16]. Several forms of the deflection angle by the Ellis wormhole have been recently derived and often used [5, 13 18]. A reason for such differences has been clarified [19, 20].

According to recent numerical calculations by Abe [14], time-symmetric demagnification parts in light curves could appear by gravitational microlensing effects of the Ellis wormhole. Is the time-symmetric demagnification evidence for the Ellis wormhole? Is is very interesting to address this question. One reason is that wormholes are inevitably related with violations of some energy conditions in physics [21]. For instance, dark energy is introduced to explain the observed accelerated expansion of the universe by means of an additional energymomentum component in the right-hand side of the Einstein equation. Furthermore, the left-hand side of the Einstein equation, equivalently the Einstein-Hilbert action, could be modified in various ways (nonlinear curvature terms, higher dimensions, and so on) inspired 
by string theory, loop quantum gravity, and so on. Because of the nonlinear nature of gravity, modifications to one (or both) side(s) of the Einstein equation might admit spacetimes significantly different from the standard Schwarzschild spacetime metric, even if the spacetime is assumed to be asymptotically flat, static, and spherically symmetric. One example is an Ellis wormhole (being an example of traversable wormholes).

Inspired by a huge number of modified theories, this brief paper assumes, in a phenomenological sense, that an asymptotically flat, static, and spherically symmetric modified spacetime could depend on the inverse distance to the power of positive $n$ in the weak-field approximation. The Schwarzschild spacetime and the Ellis wormhole correspond to $n=1$ and $n=2$, respectively. Note that Birkhoff's theorem could say that cases $n \neq 1$ might be nonvacuum, if the models were interpreted in the framework of the standard Einstein equation.

The slightly modified gravitational lensing in modified gravity theories- such as a fourth order $f(R)$ gravity theory- has attracted interests (e.g., Refs. [22 24]). It has been shown that the total magnification of the lensed images is stable and always larger than unity against a small spherical perturbation of the Schwarzschild lens [25]. This suggests that demagnifying gravitational lenses would need a significantly modified structure of the spacetime. The main purpose of this paper is to discuss demagnifying gravitational lenses due to significantly modified spacetimes.

We take the units of $G=c=1$ throughout this paper.

\section{MODIFIED SPACETIME MODEL AND MODIFIED DEFLECTION ANGLE OF LIGHT}

This paper assumes that an asymptotically flat, static, and spherically symmetric modified spacetime could depend on the inverse distance to the power of positive $n$ in the weakfield approximation. We consider light propagating through a four-dimensional spacetime, though the whole spacetime may be higher-dimensional. The four-dimensional spacetime metric is expressed as

$$
d s^{2}=-\left(1-\frac{\varepsilon_{1}}{r^{n}}\right) d t^{2}+\left(1+\frac{\varepsilon_{2}}{r^{n}}\right) d r^{2}+r^{2}\left(d \theta^{2}+\sin ^{2} \theta d \phi^{2}\right)+O\left(\varepsilon_{1}^{2}, \varepsilon_{2}^{2}, \varepsilon_{1} \varepsilon_{2}\right),
$$


where $r$ is the circumference radius and $\varepsilon_{1}$ and $\varepsilon_{2}$ are small bookkeeping parameters in the following iterative calculations. Here, $\varepsilon_{1}$ and $\varepsilon_{2}$ may be either positive or negative, respectively. A negative $\varepsilon_{1}$ and $\varepsilon_{2}$ for $n=1$ corresponds to a negative mass (in the linearized Schwarzschild metric).

For investigating light propagation, it is useful below to make a conformal transformation with a factor of $\left(1-\varepsilon_{1} / r^{n}\right)^{1 / 2}$. The null structure (such as the light propagation) is not affected by the conformal transformation. At the linear order of $\varepsilon_{1}$ and $\varepsilon_{2}$, the spacetime metric takes a simpler form:

$$
d \bar{s}^{2}=-d t^{2}+\left(1+\frac{\varepsilon}{R^{n}}\right) d R^{2}+R^{2}\left(d \theta^{2}+\sin ^{2} \theta d \phi^{2}\right)+O\left(\varepsilon^{2}\right)
$$

where $\varepsilon \equiv n \varepsilon_{1}+\varepsilon_{2}$ and

$$
R^{2} \equiv \frac{r^{2}}{\left(1-\frac{\varepsilon_{1}}{r^{n}}\right)} .
$$

Note that only one parameter $\varepsilon$ enters the conformally transformed metric.

For this metric, one can find the Lagrangian for a massless particle. Without loss of generality, we focus on the equatorial plane $\theta=\pi / 2$, since the spacetime is spherically symmetric. By using the constants of motion associated with the timelike and rotational Killing vectors, the deflection angle of light is calculated at the linear order as

$$
\begin{aligned}
\alpha & =2 \int_{R_{0}}^{\infty} \frac{d \phi(R)}{d R} d R-\pi \\
& =\frac{\varepsilon}{b^{n}} \int_{0}^{\frac{\pi}{2}} \cos ^{n} \psi d \psi+O\left(\varepsilon^{2}\right),
\end{aligned}
$$

where $R_{0}$ and $b$ denote the closest approach and the impact parameter of the light ray, respectively. This deflection angle recovers the Schwarzschild $(n=1)$ and Ellis wormhole $(n=2)$ cases. For particular cases, the above (always positive) integral factor becomes

$$
\begin{aligned}
\int_{0}^{\frac{\pi}{2}} \cos ^{n} \psi d \psi & =\frac{(n-1) ! !}{n ! !} \frac{\pi}{2} \quad(\text { even } n) \\
& =\frac{(n-1) ! !}{n ! !} \quad(\text { odd } n), \\
& =\frac{\sqrt{\pi} \frac{\Gamma\left(\frac{n+1}{2}\right)}{2}}{\Gamma\left(\frac{n+2}{2}\right)} \quad(\text { real } n>0),
\end{aligned}
$$

Henceforth, the deflection angle is denoted simply as $\alpha(b)=\bar{\varepsilon} / b^{n}$ by absorbing the numerical constant into the $\bar{\varepsilon}$ parameter. 


\section{MODIFIED LENS EQUATION AND ITS SOLUTIONS}

Under the thin-lens approximation, it is useful to consider the lens equation as [26]

$$
\beta=\frac{b}{D_{L}}-\frac{D_{L S}}{D_{S}} \alpha(b)
$$

where $\beta$ denotes the angular position of the source and $D_{L}, D_{S}, D_{L S}$ are the distances from the observer to the lens, from the observer to the source, and from the lens to the source, respectively. We wish to consider significant magnification (or demagnification), which could occur for a source in (or near) the Einstein ring. The Einstein ring is defined for $\beta=0$ [26]. If $\varepsilon<0$, Eq. (6) has no positive roots for $\beta=0$ because of the repulsive force in the particular gravity model. For $\varepsilon>0$, on the other hand, there is always a positive root corresponding to the Einstein ring. The negative $\varepsilon$ case is of less astronomical relevance. Therefore, let us consider the positive $\varepsilon$ case (causing the gravitational pull) in the following.

In units of the Einstein ring radius, Eq. (6) is rewritten as

$$
\begin{aligned}
& \hat{\beta}=\hat{\theta}-\frac{1}{\hat{\theta}^{n}} \quad(\hat{\theta}>0), \\
& \hat{\beta}=\hat{\theta}+\frac{1}{(-\hat{\theta})^{n}} \quad(\hat{\theta}<0),
\end{aligned}
$$

where $\hat{\beta} \equiv \beta / \theta_{E}$ and $\hat{\theta} \equiv \theta / \theta_{E}$ for the angular position of the image $\theta \equiv b / D_{L}$.

Let us consider two lines defined by $Y=1 / \hat{\theta}^{n}$ and $Y=\hat{\theta}-\beta$ in the $\hat{\theta}-Y$ plane. For $\hat{\theta}>0$, therefore, we have only one intersection of the two lines that corresponds to one image position. Similarly, only one image appears for $\hat{\theta}<0$.

For a general positive $n$ (e.g., $n=5$ ), it is impossible to find exact solutions for the modified lens equation. To clarify the parameter dependence, we employ analytic but approximate methods rather than numerical calculations. Furthermore, in astronomy only the significantly amplified images become detectable in gravitational microlensing. Such events occur only when a source such as a distant star crosses the Einstein ring. We thus focus on such an Einstein ring-crossing case as $\hat{\beta}<1$ in units of the Einstein ring, for which Eqs. (7) and (8) are solved in the Taylor series form with respect to $\hat{\beta}$. We obtain

$$
\begin{aligned}
& \hat{\theta}_{+}=1+\frac{1}{n+1} \hat{\beta}+\frac{1}{2} \frac{n}{(n+1)^{2}} \hat{\beta}^{2}+O\left(\hat{\beta}^{3}\right) \quad(\hat{\theta}>0), \\
& \hat{\theta}_{-}=-1+\frac{1}{n+1} \hat{\beta}-\frac{1}{2} \frac{n}{(n+1)^{2}} \hat{\beta}^{2}+O\left(\hat{\beta}^{3}\right) \quad(\hat{\theta}<0) .
\end{aligned}
$$




\section{DEMAGNIFICATION CONDITION}

The amplification factor denoted as $A$ is $|(\beta / \theta)(d \beta / d \theta)|^{-1}$, namely, the inverse Jacobian of the gravitational lens mapping between the source and image position vectors [26]. By using Eqs. (9) and (10), the amplification factor of each image, which is denoted by $A_{+}$and $A_{-}$, respectively, becomes

$$
A_{ \pm}=\frac{1}{\hat{\beta}(n+1)}+O\left(\hat{\beta}^{0}\right),
$$

where a difference between $A_{+}$and $A_{-}$appears at the next order in $\hat{\beta}$. The total amplification is thus

$$
\begin{aligned}
A_{t o t} & \equiv A_{+}+A_{-} \\
& =\frac{2}{\hat{\beta}(n+1)}+O\left(\hat{\beta}^{0}\right) .
\end{aligned}
$$

For the Schwarzschild case $(n=1), A_{\text {tot }}=1 / \hat{\beta}$. This is always larger than unity for $\hat{\beta}<1$, in concordance with the well-known fact. Demagnification of the total lensed images could occur, however, if

$$
\hat{\beta}>\frac{2}{n+1} .
$$

The larger the power $n$, the more likely the demagnification. One might guess that demagnification could be caused for a smaller $\hat{\beta}$, especially $\hat{\beta}=0$. However, this is not the case. Equation (13) suggests that the total demagnification could occur only when $\hat{\beta}$ is small but larger than the critical value $2 /(n+1)$ under a large- $n$ approximation. Note that the compatibility of the assumption $\hat{\beta}<1$ and Eq. (13) implies $n>1$. Namely, Eq. (13) becomes a better approximation as $n$ grows larger than unity.

The above argument is based on the near-zone approximation $(\hat{\beta}<1)$. For a test of the analytic result, we perform numerical calculations. We consider $n=10$, which might be one of the higher-dimensional models inspired by string theory. Equation (13) suggests that demagnification of the total lensed images could occur only for $\hat{\beta}>2 / 11=0.182$. Figure 1 shows numerical results for $n=1,2,3$, and 10 . In the case of $n=10$, the analytic result for the critical value $\hat{\beta}=2 / 11=0.182$ is in good agreement with the numerical one, $\hat{\beta}=0.187$.

Figure 2 shows numerical light curves for $n=1,2,3$, and 10. As the power $n$ is larger, time-symmetric demagnification parts in the light curves become longer in time and larger in depth. Cases of $n=3$ and 10 show maximally $\sim 10$ and $\sim 60$ percent depletion of the light, when the source position is $\hat{\beta} \sim 1.1$ and $\hat{\beta} \sim 0.7$, respectively. 

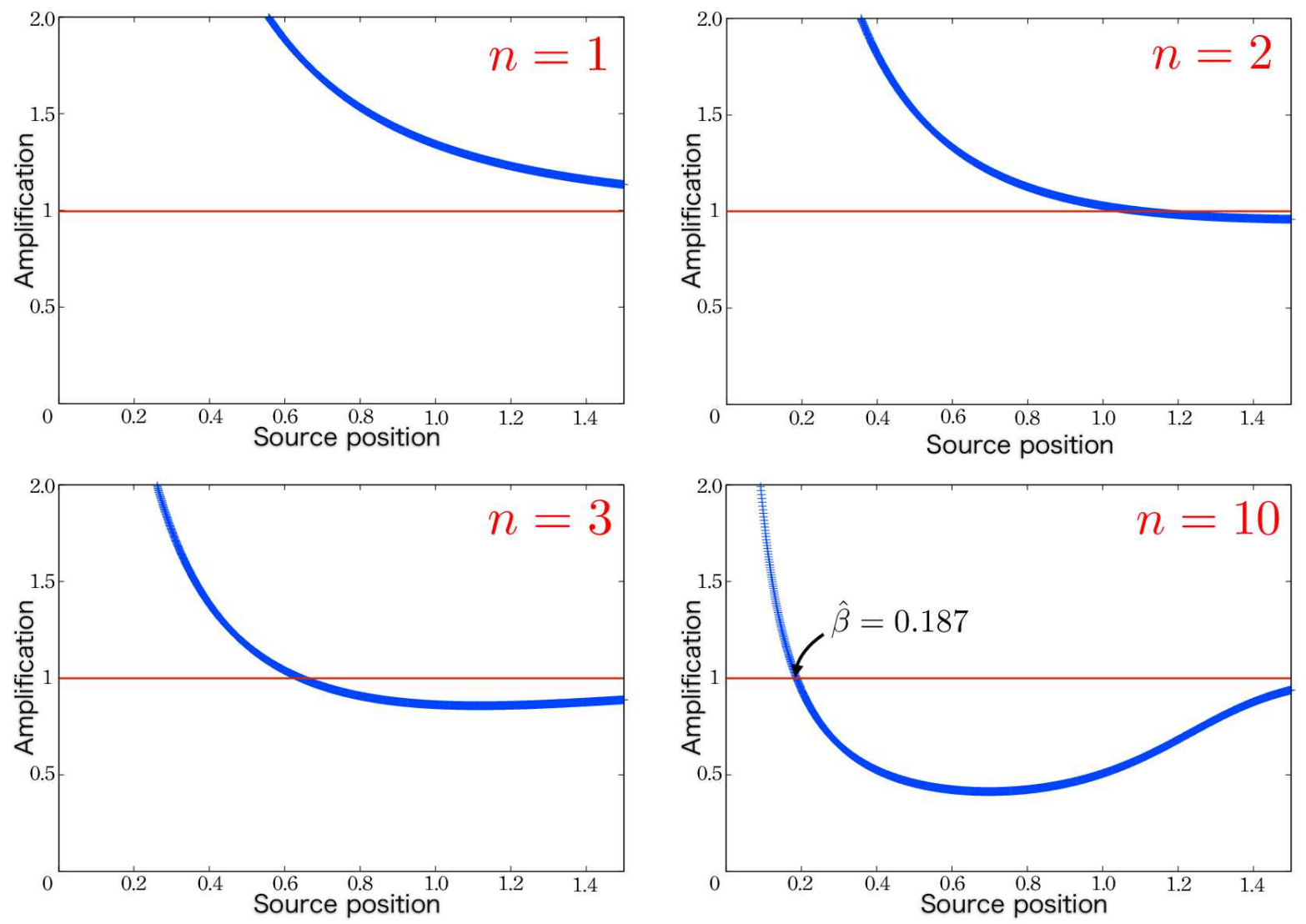

FIG. 1: Total amplification factor of the lensed images as a function of the source position $\hat{\beta}$ for $n=1,2,3$, and 10. Top left, top right, bottom left, and bottom right panels correspond to $n=1,2,3$, and 10 , respectively. In the case of $n=10$, the total amplification factor is larger than unity for $\hat{\beta}<0.187$, whereas it is smaller for $\hat{\beta}>0.187$. For convenience, a thin (red) line denotes $A_{\text {tot }}=1$.

Before closing this section, we briefly mention an effective mass. A simple application of the standard lens theory [26] suggests that the deflection $\left(\alpha=\bar{\varepsilon} / b^{n}\right)$ and magnification studied here correspond to a convergence (scaled surface-mass density) of the form

$$
\kappa(b)=\frac{\bar{\varepsilon}(1-n)}{2} \frac{1}{b^{n+1}} .
$$

For $n>1$, therefore, the effective surface-mass density of the lens object is interpreted as negative in the framework of the standard lens theory. This means that the matter (and energy) need to be exotic if $n>1$. 

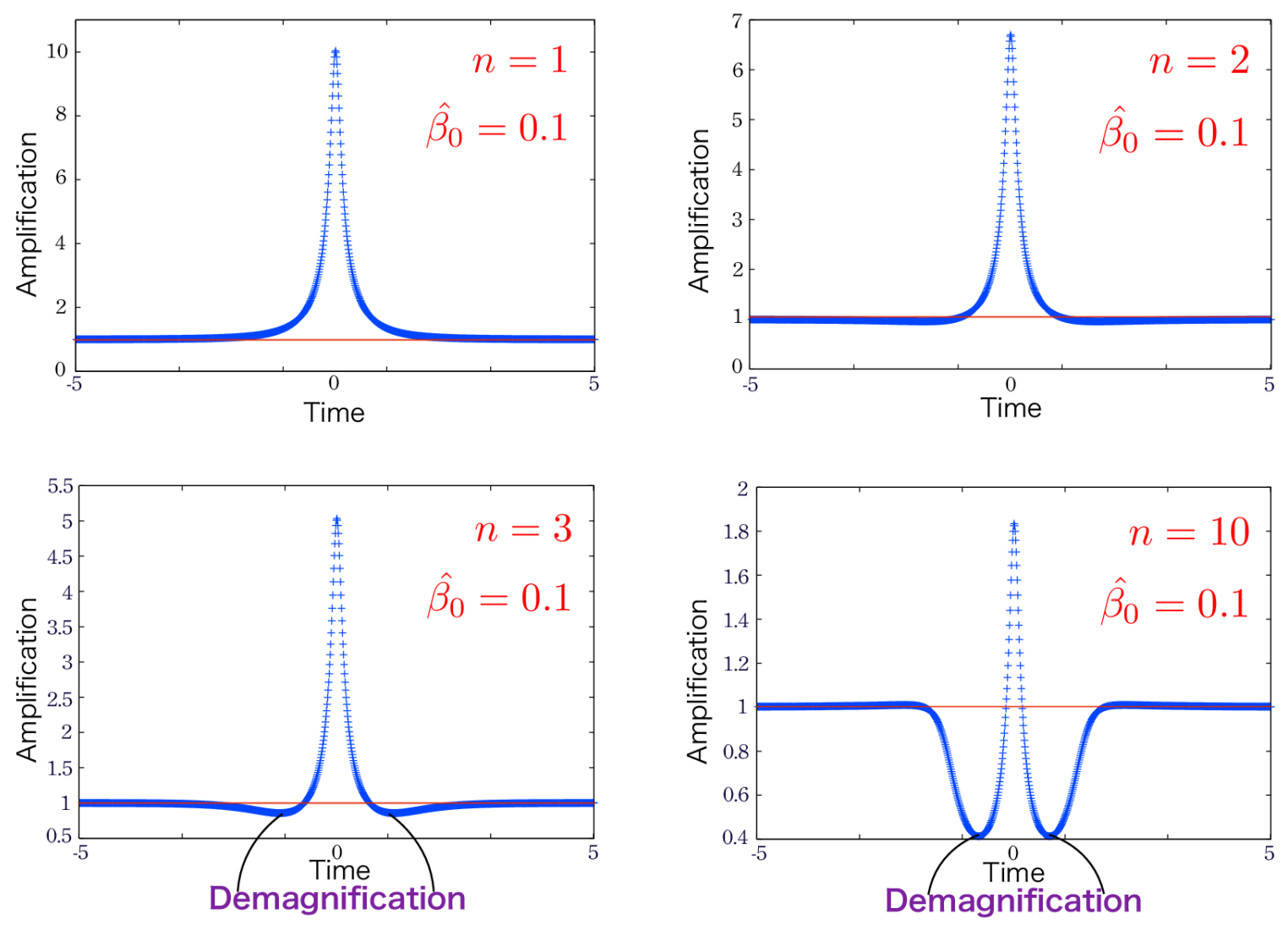

FIG. 2: Numerical light curves for the same minimum impact parameter of the light trajectory $\hat{\beta}_{0}=0.1$. The source star moves at constant speed and the source position changes as $\hat{\beta}(t)=$ $\left(\hat{\beta}_{0}^{2}+t^{2}\right)^{1 / 2}$, where time is normalized by the Einstein ring radius crossing time. Top left, top right, bottom left, and bottom right panels correspond to $n=1,2,3$, and 10, respectively. For convenience, a thin (red) line denotes $A_{\text {tot }}=1$.

\section{DISCUSSION AND CONCLUSION}

We examined a gravitational lens model inspired by modified gravity theories and exotic matter and energy. By using an asymptotically flat, static, and spherically symmetric spacetime model of which the metric depends on the inverse distance to the power of positive $n$, it was shown in the weak-field and thin-lens approximations that demagnifying gravitational lenses could appear, provided the impact parameter of light $\hat{\beta}$ and the power $n$ satisfy $\hat{\beta}>2 /(n+1)$ in units of the Einstein ring radius under a large- $n$ approximation.

Therefore, time-symmetric demagnification parts in numerical light curves by gravitational microlensing may be evidence of an Ellis wormhole, but they do not always prove 
it. Such a gravitational demagnification of light might be used for hunting a clue of exotic matter and energy that are described by an equation of state more general than the Ellis wormhole case. Examples of $n=3$ and 10 show maximally $\sim 10$ and $\sim 60$ percent depletion of the light, when the source position is $\hat{\beta} \sim 1.1$ and $\hat{\beta} \sim 0.7$, respectively. It is left to future work to perform a numerical campaign for the vast parameter space.

The above gravitational demagnification of light occurs presumably because modified lenses could act as an effectively negative (quasilocal) mass on a particular light ray (through Ricci focusing). Regarding this issue, a more rigorous formulation is needed. It would be interesting to study a relation between the model parameter $n$ and vital modified gravity theories (or matter models with an exotic equation of state) and also to make an interpretation of the parameter $n$ in the framework of the theory of general relativity.

The analytical approximate solution in this paper is obtained at the linear order of $1 / \hat{\beta}$ to discuss the total magnification. Tsukamoto and Harada [27] have studied the next order of $1 / \hat{\beta}$ to discuss the signed magnification sums, namely the difference between the amplifications of two images.

We would like to thank F. Abe, T. Harada, S. Hayward, K. Nakao, and M. Visser for useful conversations. We wish to thank N. Tsukamoto for stimulating comments on the lecture (T.K.) on the present subject at "WormHole Workshop 2012" at Rikkyo University.

[1] S. Frittelli, T. P. Kling, and E. T. Newman, Phys. Rev. D 61, 064021 (2000).

[2] K. S. Virbhadra and G. F. R. Ellis, Phys. Rev. D 62, 084003 (2000).

[3] K. S. Virbhadra and G. F. R. Ellis, Phys. Rev. D 65, 103004 (2002).

[4] E. F. Eiroa, G. E. Romero, and D. F. Torres, Phys. Rev. D 66, 024010 (2002).

[5] V. Perlick, Phys. Rev. D 69, 064017 (2004).

[6] H. G. Ellis, J. Math. Phys. 14, 104 (1973).

[7] M. S. Morris and K. S. Thorne, Am. J. Phys. 56, 395 (1988).

[8] M. S. Morris K. S. Thorne, and U. Yurtsever, Phys. Rev. Lett. 61, 1446 (1988).

[9] L. Chetouani and G. Clément, Gen. Relativ. Gravit. 16, 111 (1984).

[10] G. Clément, Int. J. Theor. Phys. 23, 335 (1984).

[11] M. Safonova, D. F. Torres, and G. E. Romero, Phys. Rev. D 65, 023001 (2001). 
[12] A. A. Shatskii, Astron. Rep. 48, 525 (2004).

[13] K. K. Nandi, Y. Z. Zhang, and A. V. Zakharov, Phys. Rev. D 74, 024020 (2006).

[14] F. Abe, Astrophys. J. 725, 787 (2010).

[15] Y. Toki, T. Kitamura, H. Asada, and F. Abe, Astrophys. J. 740, 121 (2011).

[16] N. Tsukamoto, T. Harada, K. Yajima, Phys. Rev. D 86, 104062 (2012).

[17] T. K. Dey and S. Sen, Mod. Phys. Lett. A 23, 953 (2008).

[18] A. Bhattacharya and A. A. Potapov, Mod. Phys. Lett. A 25, 2399 (2010).

[19] K. Nakajima and H. Asada, Phys. Rev. D 85, 107501 (2012).

[20] G. W. Gibbons and M. Vyska, Classical Quantum Gravity 29, 065016 (2012).

[21] M. Visser, Lorentzian Wormholes: From Einstein to Hawking (AIP, New York, 1995).

[22] S. Capozziello, V. F. Cardone, and A. Troisi, Phys. Rev. D 73, 104019 (2006).

[23] Z. Horvath, L. A. Gergely, D. Hobill, S. Capozziello, and M. De Laurentis, arXiv:1207.1823.

[24] S. Mendoza, T. Bernal, X. Hernandez, J. C. Hidalgo, and L. A. Torres, arXiv:1208.6241.

[25] H. Asada, Prog. Theor. Phys. 125, 403 (2011).

[26] P. Schneider, J. Ehlers, and E. E. Falco, Gravitational Lenses (Springer, NerYork, 1992).

[27] N. Tsukamoto and T. Harada, arXiv:1211.0380. 